\title{
Évaluation des politiques actives du marché du travail
}

\section{Introduction}

Evaluation of active labor market policies. Introduction

\section{Véronique Simonnet}

\section{(2) OpenEdition}

\section{Journals}

Édition électronique

URL : http://journals.openedition.org/travailemploi/6402

ISSN : 1775-416X

Éditeur

DARES - Ministère du Travail

\section{Édition imprimée}

Date de publication : 1 juillet 2014

Pagination : 5-14

ISSN : 0224-4365

\section{Référence électronique}

Véronique Simonnet, «Évaluation des politiques actives du marché du travail », Travail et Emploi [En

ligne], 139 | juillet-septembre 2014, mis en ligne le 01 juillet 2014, consulté le 22 septembre 2020. URL : http://journals.openedition.org/travailemploi/6402 


\title{
Évaluation des politiques actives du marché du travail Introduction
}

\author{
Véronique Simonnet $\left.{ }^{*}\right)$
}

\begin{abstract}
Depuis quarante ans maintenant, les économies dites avancées sont confrontées à un chômage de masse qui a suscité la mise en place de nombreuses politiques publiques en vue de favoriser le retour à l'emploi des chômeurs. Si ces politiques se sont développées à des rythmes et selon des modalités différents selon les pays, l'exigence d'efficacité couplée à celle de plus en plus accrue de maitrise des dépenses publiques a conduit à deux évolutions partagées sur le plan international: d'une part, le souhait de rendre «actives» les dépenses engagées au titre de la lutte contre le chômage, c'est-à-dire de mobiliser ces dernières en faveur d'actions de réinsertion sur le marché du travail plutôt que de les consacrer à la simple indemnisation (dite «passive») des chômeurs; d'autre part, la nécessité de procéder à l'évaluation méthodique des politiques mises en œuvre. Ce numéro de Travail et emploi se situe très précisément dans le prolongement de ce double objectif : élaboré à la suite d'un colloque organisé par la Direction de l'animation de la recherche, des études et des statistiques (Dares) du ministère du Travail en février $2013^{(1)}$, il entend proposer un état des lieux des connaissances existantes à la fois sur les résultats et sur les méthodes quantitatives d'évaluation des politiques actives du marché du travail (PAMT) aujourd'hui. Deux types de contribution ont été retenus : des revues de la littérature qui permettent d'aborder un large spectre des politiques conduites dans différents pays et des méthodes d'évaluation utilisées; des analyses originales où est présenté de façon détaillée un exercice d'évaluation d'une politique donnée. Avant d'en proposer ici, en introduction au numéro, un rapide parcours de lecture, nous revenons brièvement sur l'émergence progressive des politiques actives du marché du travail puis détaillons les principales méthodes statistiques permettant leur évaluation.
\end{abstract}

(*) Centre de recherche en économie de Grenoble (Creg), université Pierre-Mendès-France; Veronique.Simonnet@ upmf-grenoble.fr

(1) Pour en savoir plus sur ce colloque organisé par la Dares à Paris, les 14 et 15 février 2013, voir la page consacrée au colloque sur le site de la Dares : http://travail-emploi.gouv.fr/ etudes-recherches-statistiques-de, 76/etudes-et-recherches, 77/ manifestations-et-colloques-de-la, 99/manifestations-etcolloques-passes, 688/2013-les-politiques-actives-du, 15797. html; consultée le 27 octobre 2014.

\section{Les politiques actives du marché du travail}

Les politiques actives du marché du travail (PAMT) sont initialement et principalement destinées à aider les chômeurs à retrouver un emploi. Elles regroupent les mesures d'accompagnement personnalisé des demandeurs d'emploi et les programmes de formation à leur intention, les contrats aidés des secteurs marchand et non marchand, les aides à la création d'entreprise ainsi que les primes au retour à l'emploi des allocataires de l'aide sociale. Elles sont prônées par l'Organisation de coopération et de développement économiques (OCDE) et la Commission européenne, dans le cadre du plan d'action pour l'Europe.

Développées notamment au milieu des années cinquante en Suède, ces politiques, axées principalement sur l'offre de travail, reposent sur trois grands piliers que sont la formation professionnelle, l'orientation professionnelle et l'aide à la mobilité géographique (en plus d'un pilier plus classique d'emplois temporaires dans le secteur public ou non marchand). Considérées comme en partie responsables des deux décennies de plein-emploi que connut la Suède dans les années 1970 et 1980, alors que le taux de chômage de la Communauté européenne atteignait $10,8 \%$ en 1985 , mais surtout de l'absence de montée du chômage de longue durée en Suède, tandis que ce dernier représentait de $35 \%$ à $70 \%$ du chômage dans les pays de la Communauté, elles furent à la base des recommandations de la Stratégie de l'OCDE pour l'emploi de 1994. Et dans les années 2000, ces recommandations préconisent la mise en œuvre de programmes actifs du marché du travail afin de réduire les désincitations au travail que le versement sur une longue période de prestations élevées de chômage ou d'inactivité pouvait induire. Est ainsi visée une amélioration des perspectives de retour en emploi qui réponde à des objectifs sociaux (OCDE, 2006).

On observa un rapprochement progressif vers les politiques des trois États membres les plus actifs en la matière qu'étaient la Suède, la Finlande et le Danemark. La Suède se distinguait alors nettement des autres pays de l'Union européenne (UE) par des 
dépenses de politique active qui représentaient en 1996 près de $2,5 \%$ du produit intérieur brut (PIB). Outre la Suède, la Finlande et le Danemark, les PaysBas, l'Irlande et la Belgique avaient déjà engagé au début des années 1990 des dépenses de PAMT relativement élevées. La France, l'Allemagne et l'Italie les augmentèrent significativement plus tard dans la décennie. Ce que firent aussi le Portugal et l'Espagne, mais dans des proportions bien moindres. Au Royaume-Uni, aux Etats-Unis et au Japon, où le taux de chômage était alors relativement bas, elles restèrent plus faibles.

$\mathrm{Si}$ globalement les ressources allouées aux mesures actives se sont accrues dans tous les pays de l'Union européenne entre le milieu des années 1980 et le milieu des années 1990 (à l'exception notable du Royaume-Uni), très peu entreprirent une véritable réorientation des dépenses publiques vers des mesures actives au détriment des formules passives de garantie de revenu. La réforme du système d'aide sociale aux États-Unis en 1996 et l'adoption par l'Union européenne des lignes directrices pour l'emploi en 1997 contribuèrent alors à asseoir plus fermement une conception de la politique du marché du travail fondée sur le principe d'«obligations mutuelles» qui suppose de la part des chômeurs indemnisés qu'ils recherchent activement du travail ou participent à un programme propre à améliorer leurs perspectives d'emploi. La suppression des obstacles à l'exercice d'une activité est ainsi devenue l'une des priorités dans la plupart des pays, même si les principes d'activation ont été diversement appliqués en Europe de sorte que les résultats, en termes d'emploi, restent contrastés. Les dépenses «passives» y restent par ailleurs majoritaires.

En France, même s'il n'était pas alors présenté comme tel, le principe d'activation des dépenses de l'assurance-chômage est ancien puisqu'une allocation de formation pour les chômeurs indemnisés est instaurée dès la création de l'Unedic (Union nationale interprofessionnelle pour l'emploi dans l'industrie et le commerce), en 1958. La réforme du système d'indemnisation en 1984 ouvre la voie à de nouvelles mesures «actives» avec, entre autres, le mécanisme «d'intéressement» (1986) qui permet le cumul d'un salaire d'activité réduite et d'une fraction de l'indemnisation, les conventions de conversion (1987), les allocations de formation reclassement (1988), les conventions de coopération (1994) ou l'allocation de remplacement pour l'emploi en 1995. Alors que la nouvelle réforme du système d'indemnisation en 1992, instaurant l'allocation unique dégressive, repose encore sur le principe de diminution régulière du montant des prestations dans le but d'accroitre l'incitation au retour à l'emploi, la nouvelle convention Unedic de 2001 retient une autre forme d'activation : elle privilégie l'accompagnement des demandeurs d'emploi à l'incitation supposée de la dégressivité des allocations, en adoptant un plan d'aide au retour à l'emploi (Pare) qui permet un suivi des chômeurs sans diminution des indemnités reçues au cours du temps.

Ce qui relève de la notion polysémique de dépense publique active a concerné aussi très tôt l'aide sociale, comme le rappelle le Conseil de l'emploi, des revenus et de la cohésion sociale (CERC, 2005). Ainsi, l'orientation vers une politique active a été retenue dès la création du revenu minimum d'insertion (RMI) en 1988. Le législateur pose le principe, non seulement d'une allocation d'aide sociale permettant de disposer d'un revenu minimal, mais aussi d'un instrument visant à permettre l'insertion économique et sociale des allocataires. La loi de 1988 indique en effet que : «Toute personne [...] qui s'engage à participer aux actions ou activités définies avec elle, nécessaires à son insertion sociale ou professionnelle, a droit, [...] à un revenu minimum d'insertion » (loi n ${ }^{\circ} 88-1088$ du $1{ }^{\text {er }}$ décembre 1988 relative au revenu minimum d'insertion, article 2). Le principe est réaffirmé lors de la mise en place du revenu de solidarité active (RSA), en 2009, en remplacement du RMI, de l'allocation pour parents isolés (API) et des dispositifs associés d'aide financière à la reprise d'emploi. Le RSA diffère de l'intéressement qui prévalait depuis 1988 en proposant un cumul désormais pérenne et plus avantageux de l'allocation avec une partie des revenus d'activité, induisant en théorie une incitation durable à la reprise d'un emploi.

Les mesures d' «activation» reposent sur le principe d'«obligations mutuelles» qui exige des chômeurs indemnisés qu'ils recherchent activement du travail et/ou améliorent leur «employabilité» en contrepartie d'allocations et services aidant au retour en emploi. Cette stratégie a pour but de mobiliser davantage les allocataires et le service public de l'emploi. Les chômeurs sont par exemple tenus d'avoir des entretiens approfondis avec des conseillers spécialisés, de répondre aux offres d'emploi proposées par ces derniers, d'accepter les emplois qui leur sont présentés, de prendre part à l'élaboration de plans d'action individuels, de suivre une formation ou de participer à des dispositifs de création d'emplois. Les programmes d'activation ont pour cible principale les personnes qui bénéficient (ou qui demandent à bénéficier) de revenus de remplacement dont le versement est soumis à la condition d'être disponible pour travailler. Cependant, face à la hausse tendancielle du nombre global d'allocataires de prestations sociales, ils ont été élargis aux personnes qui n'étaient pas considérées jusque-là comme dépourvues d'emploi. Ils concernent désormais la majeure partie des chômeurs indemnisés, auxquels s'ajoutent souvent les parents isolés et les allocataires de l'aide sociale.

Ces programmes sont supposés avoir un effet sensible sur les résultats en termes d'emploi dans le 
cas des régimes de prestations de longue durée. Ils doivent contribuer à accroître le recours des participants à des services qui vont les aider à être plus performants dans leur recherche d'emploi et/ou à améliorer leurs compétences. Ils doivent conduire à des résultats quasi similaires à ceux obtenus en réduisant uniquement le montant des prestations (pour inciter à une intensification de la recherche d'emploi) mais tout en conservant un niveau de protection sociale plus élevé. De plus, ces dispositifs doivent pouvoir s'adresser à des participants peu familiarisés avec les services de placement, de conseil et de formation, et qui, en l'absence de réglementation, n'y recourraient pas et ne pourraient donc en tirer profit. Enfin, étant donné le «coût» associé à l'obligation d'y participer(2), certaines personnes qui pourraient prétendre à des prestations n'en font pas la demande, tandis que d'autres qui en bénéficient déjà vont rapidement prendre un emploi ou renoncer à leurs droits plus tôt qu'elles ne l'auraient fait auparavant. Cependant et afin d'apporter un soutien nécessaire aux demandeurs d'emploi qui en ont besoin, les programmes d'activation sont censés ne pas imposer d'obligations trop contraignantes qui décourageraient toute demande d'assistance. C'est pourquoi les stratégies d'activation des pays de l'OCDE tendent pour la plupart à privilégier la logique des «services en faveur de l'emploi» et évitent de recourir à l'obligation de travail en échange des prestations (OCDE, 2005).

La nomenclature «Politiques du marché du travail»(PMT) élaborée en 1996 par Eurostat permet de suivre les dépenses ciblées en faveur du marché du travail dans les différents États membres, et notamment en France (voir Garoche, Roguet, 2014, encadré 3, p. 8). L'évolution des dépenses actives du marché du travail en France a ainsi été publiée pour la première fois par l'Institut national de la statistique et des études économiques (Insee) en 2009 et retraçait alors la période 2000-2007 (Roguet, Schreiber, 2009). Les plus récentes publications nous permettent d'étendre cette période jusqu'à 2011 pour l'ensemble des dispositifs et 2013 pour certains contrats aidés (BAHU, 2014; Bayardin, Fendrich, 2014; Dares, 2014; Garoche, Roguet, 2014).

En 2008, les dépenses pour les politiques en faveur du marché du travail représentaient en France $2 \%$ du PIB. Sachant que les pays de 1'Union européenne à $27 \mathrm{y}$ ont consacré en moyenne cette même année $1,6 \%$ de leur PIB, ce pourcentage place la France au septième rang derrière la Belgique, l'Espagne, le Danemark, les Pays-Bas, la Finlande et l'Irlande. Il est néanmoins le plus faible observé

(2) Les entretiens plus nombreux avec des conseillers spécialisés, les injonctions plus ou moins fortes à suivre une formation et l'obligation de répondre à certaines offres d'emploi ou d'accepter certains emplois peuvent être perçus comme autant de contraintes. pour la France depuis le début de la dernière décennie, ce qui résulte d'une amélioration sensible de la situation sur le marché du travail durant les années 2006-2007 (le point le plus haut avait été atteint en 2003 avec 2,8 points de PIB). Les dépenses d'indemnisation au titre du chômage constituent $57 \%$ de ces dépenses en 2007-2008, loin devant les aides à l'emploi (14\%), la formation professionnelle des demandeurs d'emploi (15\%) et les moyens consacrés au service public de l'emploi (11\%).

$\mathrm{Au}$ sein des dépenses actives, les aides à l'emploi non marchand représentent $40 \%$ au début des années 2000. Les contrats aidés - en particulier dans le secteur non marchand - constituent alors un des outils privilégiés pour faire face aux ralentissements conjoncturels de l'activité, faisant écho à l'expansion spectaculaire de l'emploi public en Suède, entre 1970 et 1985, en réponse aux chocs pétroliers. Avec l'extinction progressive des emplois jeunes et les recentrages successifs des autres contrats sur les publics les plus en difficulté, les aides à l'emploi dans le secteur non marchand ont fortement diminué jusqu'en 2005. À partir de 2006, les contrats aidés du Plan de cohésion sociale prennent le relais, entraînant un léger rebond des dépenses. À la suite du sévère retournement de l'activité lié à la crise financière de 2007, ces contrats connaissent une forte progression en 2009 et 2010 avant de reculer de nouveau, et fortement, en 2012, les dépenses atteignant alors leur plus bas niveau depuis plus de dix ans.

Les aides à l'emploi concurrentiel représentaient, quant à elles, $18 \%$ des dépenses actives en 2000 . Ce poids a varié tout au long de la décennie. En 2013, bien qu'en légère augmentation, les contrats aidés hors alternance du secteur marchand concernent des effectifs sensiblement plus faibles que ceux observés jusqu'en 2010.

C'est la formation professionnelle qui occupe désormais une place prédominante dans les politiques actives, avec une forte croissance depuis la crise de 2007. Les dépenses afférentes représentent $39 \%$ des dépenses actives en 2011. Cet essor marque le rôle accru des conseils régionaux qui, prenant le relais de l'État du fait de la décentralisation, ont progressivement développé leurs politiques de formation en faveur des demandeurs d'emploi. Cette évolution, qui incite les régions à s'impliquer dans la lutte contre le chômage de longue durée afin de rapprocher l'aide sociale de la politique de l'emploi, suit les expériences menées au Danemark, puis en Suède, au cours des années 1990.

En parallèle, les aides aux chômeurs créateurs ou repreneurs d'entreprise, qui consistent principalement en une exemption de cotisations sociales la première année d'activité, progressent sensiblement, occupant une part croissante des dépenses actives depuis 2006 . 


\section{Les évaluations statistiques des politiques actives}

Aider les demandeurs d'emploi en leur assurant une aide et un suivi intensifs, des prestations généreuses et parfois même des compensations financières à l'exercice d'une activité réduite, peut peser sur les budgets publics. Dans un contexte de rationalisation des dépenses, la promotion de travaux d'évaluation quantitative des programmes ou dispositifs de politiques publiques du marché du travail a été engagée. Ces travaux ont pour finalité de vérifier si les objectifs assignés aux programmes sont bien atteints, c'est-à-dire de voir s'ils améliorent la situation des individus bénéficiaires sur le marché du travail. Ils doivent pour cela mesurer les résultats obtenus et tenter d'expliquer les écarts observés entre effets attendus et mesurés. L'évaluation d'un dispositif d'action publique se distingue en ce sens de son contrôle et de son pilotage, qui consistent à s'assurer, tout au long du processus, de la conformité du dispositif engagé aux normes de référence. On sépare ainsi l'évaluation de processus qui se penche sur la mise en œuvre de la politique de l'évaluation d'impact qui vise à apprécier, de la manière la plus objective possible, les effets d'un programme sur la société (Gomel, Serverin, 2013). Ce sont aux évaluations d'impact que se consacre spécifiquement ce numéro.

L'évaluation des politiques publiques en France $\mathrm{a}$, au cours des deux dernières décennies, été marquée par des tâtonnements institutionnels, qui peuvent expliquer, du moins en partie, son absence du débat public (PerRet, 2008). Cela étant, récemment, la révision de juillet 2008 a inscrit la notion d'évaluation des politiques publiques au sein même de la Constitution et la modernisation de l'action publique annoncée en décembre 2012 prévoit que «toutes les politiques publiques, sur l'ensemble du quinquennat, feront l'objet d'une évaluation». En pratique, les évaluations sont en partie prises en charge par la Cour des comptes, institution de contrôle plus que d'évaluation. En outre, les travaux d'évaluation d'impact montent en puissance dans le milieu académique, notamment dans les domaines de l'emploi et de l'éducation. Une « culture de l'évaluation» semble ainsi se développer en France, qui rejoint les États-Unis, le Royaume-Uni ou encore les pays d'Europe du Nord où ces études sont plus anciennes.

Dans tous ces pays en effet, l'évaluation des politiques actives du marché du travail occupe une place importante. Les programmes de formation professionnelle destinés aux demandeurs d'emploi ont été très tôt et largement étudiés (voir par exemple, Gritz, 1993; Bonnal et al., 1997; GerFin, LeChNER, 2002 et SiANESI, 2004) tout comme les politiques basées sur les incitations financières à la reprise d'emploi (voir par exemple, EISSA,
LieBMan, 1996; CARD et al., 2005; FranCESCONI et al., 2009). Ces travaux convergent pour pointer que les programmes ayant le contenu en formation professionnelle le plus important sont les plus efficaces en termes d'insertion (FoUGÈre et al., 2000), que les mères isolées sont particulièrement sensibles aux incitations financières liées au retour à l'emploi (Meyer, Rosenbaum, 2001) et que l'effet des dispositifs d'aide au retour à l'emploi diffère sensiblement selon les publics concernés (HECKMAN et al., 1999). Plus récemment, l'intérêt s'est porté sur l'accompagnement des demandeurs d'emploi dans leur démarche de recherche d'emploi. BoONE et al. (2007) démontrent qu'un suivi et des conseils personnalisés dans la recherche d'emploi, lorsque celle-ci peut être contrôlée et influencée à un coût raisonnable, est plus efficace que la simple dégressivité des allocations-chômage, ce que BLUNDELL et al. (2004) et CRÉPON et al. (2005) confirment sur données britanniques et françaises.

En France, les travaux de Dormont et al. (2001) montrent que la trop rapide dégressivité des allocations-chômage instaurée en France en 1992 a conduit à un ralentissement des retours à l'emploi, augurant du changement opéré en 2001 dans le système d'indemnisation. Ceux de Brodaty et al. (2001) ou encore Brodaty (2007) mettent en avant que les dispositifs les plus formateurs produisent généralement les impacts moyens les plus importants, validant ainsi l'orientation prise par les pouvoirs publics dans les années 2000 en direction des dispositifs de formation professionnelle des demandeurs d'emploi. Plus récemment, BeHAGHeL et al. (2009) et FoUgĖRE et al. (2010) ont confirmé que les mesures d'accompagnement des demandeurs d'emploi expérimentées en France avaient contribué à augmenter la reprise d'emploi des bénéficiaires, confortant le renforcement de ces mesures au sein des politiques actives du marché du travail depuis la mise en place du plan d'aide au retour à l'emploi instauré en juillet 2001. À l'inverse, la décision de généralisation du RSA à l'ensemble du territoire, avant même que les résultats de l'expérimentation du dispositif ne soient connus, souligne que les travaux d'évaluation n'ont pas toujours la portée attendue.

Tous ces travaux mobilisent des méthodes statistiques ou économétriques d'évaluation qui se sont développées ces trente dernières années. Les méthodes d'évaluation d'un programme ou d'une politique publique cherchent à répondre à la question fondamentale suivante: quelle aurait été la situation économique des individus bénéficiaires en l'absence de programme ? La réponse à cette question cherche à mettre en évidence l'effet moyen du programme sur les bénéficiaires (Blundell, CoSTADIAS, 2008).

L'effet causal d'un programme est défini pour chaque individu par la différence entre ce que serait 
sa situation s'il y participait et ce qu'elle serait s'il n'y participait pas. Comme l'individu ne peut être dans deux situations à la fois (participant et non participant), l'effet causal est inobservable. Il est de plus individuel car il peut y avoir un effet différent du programme sur chaque participant. On peut cependant rechercher l'effet moyen du programme sur la population en comparant la situation moyenne des bénéficiaires à la situation moyenne d'un groupe de non-bénéficiaires dont la situation est supposée très proche de celle qu'auraient connue les bénéficiaires s'ils n'avaient pas participé au programme. La constitution de ce groupe de «contrôle» ou contrefactuel est loin d'être évidente. Les problèmes qu'elle soulève ont été décrits initialement par Donald B. RuBIN (1974) avec un vocabulaire qui emprunte beaucoup au champ de l'expérimentation médicale dont il est issu. On parle alors de groupe « traité» au sujet des bénéficiaires et de l'effet causal du traitement.

Les méthodes qui suivent proposent différents modes de constitution du fameux groupe de contrôle, qui dépendent du contexte et des données disponibles. Nous en présentons un aperçu rapide qui sera aussi l'occasion de présenter les articles qui composent ce numéro à travers leur mise en œuvre de ces méthodes traditionnelles d'évaluation.

\section{Les expériences contrôlées}

Le cadre souvent cité comme idéal pour l'évaluation d'un dispositif est l'expérimentation aléatoire qui suppose que la conception et la mise en œuvre d'une politique soient pensées simultanément à son évaluation de sorte que puissent être constitués, en amont, deux groupes parfaitement identiques au sein de la population des individus éligibles au dispositif présenté : l'un auquel le dispositif sera effectivement proposé, l'autre auquel le dispositif ne sera pas directement proposé. Cette démarche, qui s'inspire des méthodes utilisées depuis longtemps dans le milieu médical(3), revient à tirer au sort les bénéficiaires et à s'assurer qu'en moyenne, bénéficiaires et non-bénéficiaires ont des caractéristiques semblables. Il n'y a plus ensuite qu'à comparer la situation moyenne des deux groupes une fois le dispositif mis en œuvre pour évaluer son impact.

Aujourd'hui, les expérimentations aléatoires occupent une place de plus en plus importante en sciences sociales. Leur grand avantage est leur apparente simplicité : il suffit de répartir aléatoirement les personnes en deux groupes, de donner le traitement à l'un des groupes, et d'observer ensuite si son comportement diffère de l'autre groupe pour savoir si le traitement a un impact significatif ou

(3) $C f$. notamment, le numéro 135 que la revue Travail et emploi a consacré aux expérimentations de terrain des politiques du travail et de l'emploi. non. Une limite claire est cependant qu'il n'est pas toujours possible de procéder à un tirage au sort pour déterminer qui sera traité, l'impossibilité pouvant être due à des raisons éthiques ou à l'organisation pratique du tirage au sort. À défaut, on peut utiliser des dispositifs "quasi expérimentaux» appelés aussi «expériences naturelles» par opposition aux expérimentations qui sont dites «expériences contrôlées». On utilise dans ce cas le fait que certaines personnes sont exclues ou partiellement exclues du bénéfice du programme évalué soit parce que les législations qui s'appliquent à elles sont différentes, soit parce que certaines de leurs caractéristiques personnelles limitent leur accès au dispositif. On fait alors l'hypothèse que les différences ainsi constatées, différences de législation ou de caractéristiques individuelles (considérées alors comme des variables instrumentales), expliquent la non-participation ou la moins forte participation au programme évalué mais sont indépendantes des variables qui ont une incidence sur le résultat (GIVORD, 2010).

La plupart des travaux d'évaluation des programmes d'accompagnement des demandeurs d'emploi mis en œuvre en Europe dans les années 1990 et 2000 ont été réalisés dans le cadre d'expérimentations contrôlées comme nous l'enseigne dans ce numéro l'article de Gwenn Parent et Olivia Sautory qui en retrace les principaux résultats. Parmi eux, l'évaluation de deux programmes d'accompagnement, expérimentés pour la première fois en France en 2007, montre que l'accompagnement renforcé effectué par le service public est plus efficace que celui réalisé par le secteur privé même si la coexistence des deux accompagnements a pu susciter une émulation positive entre les prestataires (Behaghel et al., 2009).

L'expérimentation aléatoire permet de plus de traiter le problème de la non-participation au programme de personnes y ayant été initialement invitées. L'idée est alors de supposer, comme les deux groupes («traités» et «contrôle») sont identiques, qu'il aurait été observé la même défection au programme de la part des individus du groupe de contrôle si ces derniers avaient été choisis pour participer. En comparant la situation de l'ensemble des personnes sollicitées pour participer au programme (qu'elles y participent réellement ou non) à l'ensemble des personnes non sollicitées pour y participer, on annule le biais résultant de la non-participation de certains allocataires étant donné qu'il est identique dans les deux groupes.

Les évaluations des dispositifs de contrôle de l'effectivité des recherches d'emploi opérées par les chômeurs indemnisés aux États-Unis et en Europe - notons que ce type de dispositif n'a encore jamais été évalué en France - ont pratiquement toutes eu recours à des expérimentations aléatoires. L'article de Gwenn Parent qui présente dans ce numéro la 
mise en œuvre des politiques de contrôle et sanctions en Europe détaille les résultats des évaluations des programmes les plus précurseurs au niveau européen que sont le "Jobseeker's Allowance», mis en place au Royaume-Uni en 1996 et le «Counselling and Monitoring ", appliqué aux Pays-Bas en 1998 tout comme ceux, plus récents, des expérimentations sociales menées en Belgique et au Danemark dans le milieu des années 2000.

\section{La régression par discontinuité}

La méthode dite de «régression par discontinuité» est fondée sur le fait que, pour de nombreux dispositifs, il existe des seuils qui permettent d'en limiter l'accès aux bénéficiaires potentiels. Il peut notamment s'agir du seuil de revenus pour la perception d'une allocation ou alors d'âge pour bénéficier d'un minimum social ou accéder à un dispositif d'aide à la création d'entreprise ou encore de conditions portant sur le nombre et l'âge des enfants pour bénéficier d'une allocation parentale par exemple. Ainsi, la probabilité de bénéficier de ces dispositifs est discontinue, parfois fortement. Or, les personnes situées juste au-dessous du seuil et celles situées juste au-dessus sont en général très proches pour tout un ensemble de caractéristiques et on peut considérer que les différences d'âge ou de revenus observées entre ces personnes sont trop faibles pour qu'elles aient un impact significatif sur leur situation après qu'elles ont ou non participé au programme. On peut donc les comparer, après la mise en place du dispositif, pour en évaluer l'impact sans craindre la présence d'effets de population trop importants. Si le principe de la méthode est simple, elle requiert cependant de détenir suffisamment d'observations de part et d'autre du seuil défini par le dispositif, dans une fenêtre qu'il reste à choisir mais qui doit être relativement restreinte. Il s'agit de s'assurer que l'estimateur retenu permette de mesurer des effets mêmes réduits et que la mesure statistique obtenue sur cette population restreinte soit fiable.

Récemment, cette méthode a été utilisée pour évaluer l'effet d'un allongement de la durée d'indemnisation du chômage sur la durée même du chômage (LALIVE, 2008). En 1988, certaines régions d'Autriche, confrontées à la baisse d'activité des industries de l'acier, ont augmenté considérablement la durée d'indemnisation du chômage des personnes de 50 ans et plus. En comparant les durées de chômage observées pour les personnes de moins de 50 ans et celles de 50 ans et plus, après la réforme, Rafael LALIVE constate un écart d'environ quinze semaines (les personnes entre 46 et 49 ans ayant des durées moyennes de chômage de l'ordre de treize semaines tandis que celles âgées de 50 à 54 ans des durées moyennes proches de vingt-huit semaines). La durée d'indemnisation du chômage semble donc avoir un effet significatif sur la durée de chômage.

\section{L'appariement sur les caractéristiques observables}

Une autre méthode couramment employée pour l'évaluation est la méthode d' «appariement» ou «matching» (Imbens, 2000; CALIEndo, Kopeinig, 2005). Pour chaque individu bénéficiant du dispositif, il s'agit de trouver un individu non bénéficiaire ayant des caractéristiques observables similaires - sexe, âge, diplôme, historique sur le marché du travail, etc. - et de comparer leurs situations respectives. L'hypothèse est alors faite que, conditionnellement à ces caractéristiques, la propriété d'indépendance entre la participation au programme et le résultat est vérifiée. Cette propriété d'indépendance suppose la prise en compte d'un nombre important de variables. Pour cette raison, l'appariement peut être difficile en pratique. En effet, pour certains individus traités, on peut n'observer aucun individu non traité ayant exactement les mêmes caractéristiques. Le problème du nombre de variables pertinentes à retenir est cependant fortement réduit par une propriété mise en évidence par Paul R. Rosenbaum et Donald B. Rubin (1983). Ces derniers montrent qu'il suffit d'apparier traités et non traités en fonction de leur score de propension (ou probabilité d'être traité), lequel constitue un résumé unidimensionnel de l'ensemble des variables. L'estimateur peut de plus être amélioré en prenant une moyenne pondérée des observations de l'échantillon de contrôle (la population des individus non traités). Chaque individu non traité participe ainsi à la construction du contrefactuel d'un individu traité avec une importance qui varie selon la distance entre son score et celui de l'individu considéré (HECKMAN et al., 1997).

Cette méthode est employée dans ce numéro par Gesin Stephan pour évaluer l'effet de la mise en place de deux types de contrats aidés en Allemagne à la fin des années 1990. Ces contrats, qui prévoient une subvention de la part de l'État à hauteur de $50 \%$ du salaire proposé, sont destinés aux chômeurs relativement éloignés de l'emploi ou nécessitant une formation professionnelle spécifique. Son utilisation d'une base de données administrative particulièrement riche, détaillant à la fois les caractéristiques sociodémographiques des chômeurs, leur parcours sur le marché du travail et les caractéristiques de l'emploi retrouvé et de l'entreprise offrant cet emploi, lui permet d'apparier au mieux les chômeurs de longue durée ayant finalement obtenu un contrat aidé avec ceux, semblables, ayant obtenu un contrat de droit commun dans le même secteur. L'auteure peut ainsi évaluer l'efficacité du dispositif selon le secteur d'activité de l'entreprise qui y recourt et voir si des pratiques abusives apparaissent dans certains secteurs.

La non-prise en compte du processus d'embauche de ces chômeurs ni du rôle du service public de l'emploi dans le choix des chômeurs de 
passer par un contrat aidé plutôt que par un contrat de droit commun compromet cependant quelque peu les chances d'éliminer totalement le biais de sélection. Si la méthode de l'appariement réduit les erreurs que l'on peut commettre en faisant une comparaison simple entre traités et non traités, on ne corrige pas la sélection dite «inobservable». Par exemple, dans une population de chômeurs, certains demandeurs d'emploi peuvent entreprendre plus de démarches pour retrouver rapidement un emploi tandis que d'autres, plus découragés, peuvent être moins motivés. Aucun fichier statistique ne contient ces informations «inobservables». Elles sont pourtant très importantes car elles conditionnent à la fois l'entrée dans un programme et la capacité à sortir spontanément du chômage vers l'emploi. Elles vont donc fortement influencer le résultat en venant biaiser les comparaisons entre participants et nonparticipants. Il ne s'agit pas là d'un problème de second ordre que l'on pourrait négliger en première approximation. HeCKMAN et al. (1997) montrent que les biais résiduels avec la méthode de l'appariement sont en général de l'ordre de grandeur de l'effet du programme lui-même.

\section{La méthode des doubles différences}

Pour éliminer le biais lié à l'hétérogénéité inobservée, on peut combiner la méthode de l'appariement avec la méthode de différences de différences. La terminologie différences de différences traduit le fait que l'on considère la variation dans le temps de la variable de résultat, ce qui constitue la première différence, que l'on compare entre les traités et les non-traités, ce qui en implique une seconde. On prend comme référence une période qui précède l'introduction du programme pour laquelle on retient les principales caractéristiques de l'ensemble des individus, ce qui permettra de constituer a posteriori le groupe traité et le groupe non traité éligible. On réitère cette collecte d'informations après l'introduction du programme, une fois que les bénéficiaires et les non-bénéficiaires sont connus. On calcule la différence pour chacun des groupes avant et après l'introduction du programme et, enfin, on soustrait à la différence observée pour le groupe traité celle qui a été obtenue pour le groupe de contrôle. On obtient alors la propriété d'indépendance conditionnellement à des observables pour des évolutions des variables de résultat. HECKMAN et al. (1998) proposent ainsi une généralisation de l'estimateur par différences de différences.

En comparant les situations des bénéficiaires et des non-bénéficiaires en évolution et non en statique, on élimine le biais de sélection lié à l'hétérogénéité inobservée qui doit être présent aussi bien avant la réforme qu'après. En comparant de plus l'évolution des situations des bénéficiaires et des non-bénéficiaires sur la même période, on neutralise aussi l'effet de la conjoncture. La démarche n'est cependant valide que si l'on suppose que les deux groupes auraient eu les mêmes évolutions en l'absence de réforme.

Dans ce numéro, Andreas Moczall retient la méthode de double différence avec appariement selon le score de propension pour évaluer les possibles effets d'aubaine et de substitution associés à la mise en place de contrats aidés pour l'emploi de bénéficiaires de l'aide sociale particulièrement défavorisés, en Allemagne. L'effet d'aubaine intervient lorsque des entreprises recourent à un contrat aidé alors même qu'elles auraient embauché si la subvention n'avait pas été proposée. On parle d'effet de substitution lorsque l'emploi subventionné privilégie une certaine catégorie de travailleurs au détriment d'autres qui auraient été embauchés si le coût du travail associé aux premiers n'avait pas été abaissé.

Pour mesurer l'ampleur de ces effets, l'auteur compare l'évolution de l'emploi régulier (ou non subventionné) dans les entreprises recourant au dispositif et dans les autres. Il ne met en évidence, grâce à des données administratives couvrant l'ensemble des entreprises allemandes, ni d'effet d'aubaine ni d'effet de substitution pour le programme «JobPerspektive» mis en place en Allemagne en 2007-2010. Et il constate même une augmentation de l'emploi régulier pour les entreprises ayant pu bénéficier du dispositif.

Les méthodes présentées jusqu'ici s'attachent à caractériser la situation des bénéficiaires à un moment donné du temps ou à deux moments précis représentant la période avant et après la mise en œuvre du dispositif. Lorsque l'on veut comparer les transitions de chômeurs vers l'emploi sachant que ces transitions peuvent intervenir à des moments différents du temps et que le recours à un dispositif peut, lui aussi, intervenir après un temps variable passé au chômage, on fait alors appel aux modèles de durée qui ont l'avantage de pouvoir tenir compte de la séquentialité et de la durée des événements et du fait que certains individus ne connaîtront pas de transitions sur la période observée.

\section{La méthode du "timing of events"}

Les modèles de durée ont été particulièrement employés pour évaluer si la pratique d'une activité réduite peut aider les chômeurs à retrouver plus rapidement un emploi. L'expérience consistant à permettre à certains chômeurs de cumuler une partie des indemnités chômage avec les revenus d'une activité réduite a été tentée en Allemagne, Autriche, Belgique, Espagne, Finlande, France, Grèce, Luxembourg et Suisse. De nombreuses évaluations ont été menées pour évaluer si l'effet bénéfique (ou effet tremplin) associé à la pratique d'une activité réduite, à savoir l'augmentation de l'expérience professionnelle et le signal d'une plus forte employabilité, pouvait dépasser l'effet d'enfermement que suscite l'activité réduite, limitant 
l'effort de recherche d'un emploi régulier une fois l'activité réduite entreprise.

Les transitions du chômage vers l'emploi des chômeurs selon qu'ils ont ou non accepté un emploi en activité réduite sont alors comparées. En comparant simplement les parcours des «traités» et des «contrôle», on mesure cependant à la fois l'effet de l'activité réduite sur le retour à l'emploi régulier mais aussi l'effet des caractéristiques observées et/ou inobservées des deux groupes (en termes d'employabilité, par exemple). Pour résoudre ce problème, la méthode dite du "timing of events » ou, plus explicitement, de l'échelonnement dans le temps des événements exploite le fait que l'hétérogénéité inobservée affecte la transition vers l'emploi régulier tout le temps de la période de chômage alors que l'activité réduite (le traitement) n'affecte la transition qu'à partir du moment où elle démarre. Du fait de cette discontinuité, on peut isoler l'effet traitement de l'activité réduite de l'effet sélection dû aux caractéristiques particulières des individus qui passent par une activité réduite sans imposer de restriction d'exclusion (ABBRING, VAN DEN BERG, 2003).

À partir de cette méthode et à l'aide de données administratives particulièrement riches, Florent Fremiggacci et Antoine Terracol montrent, dans ce numéro, que la pratique d'une activité réduite en France occasionne au départ un fort effet d'enfermement, puis un effet tremplin tel, que l'effet tremplin finit par l'emporter. Les individus pour lesquels le montant cumulé de salaire et d'indemnité est relativement faible recourent à des activités réduites de meilleure qualité, ce qui améliore sensiblement leurs transitions vers l'emploi régulier.

\section{Les articles du numéro}

Les travaux présentés dans ce numéro nous permettent d'avoir un aperçu d'une grande partie des politiques actives du marché du travail entreprises ces trente dernières années en France et en Europe. L'article de Fremiggacci et Terracol s'intéresse à l'une des premières réformes du système d'assurance-chômage en France, qui s'est attachée à augmenter l'employabilité des chômeurs et à ainsi tenter d'accroître leur participation au marché du travail en permettant, dès 1986, le cumul partiel des revenus d'une activité réduite avec les indemnités chômage. Leurs résultats nous enseignent que la mise en place d'un dispositif trop généreux, c'està-dire autorisant un cumul relativement important des revenus d'activité avec les indemnités perçues, serait peu efficace dans le sens où il n'inciterait pas les demandeurs d'emploi à sélectionner les activités réduites les plus susceptibles d'accélérer leur transition vers l'emploi durable. Ils suggèrent de plus de cibler ce dispositif sur les chômeurs les plus éloignés de l'emploi pour lesquels l'effet tremplin recherché est le plus fort.

Les articles de Stephan et Moczall étudient des dispositifs de subventions à l'emploi mis en place avant, puis dans le cadre des réformes «Hartz» du marché du travail allemand. Les contrats aidés présentés par Stephan ont été mis en œuvre entre 1998 et 2003 à destination des chômeurs relativement éloignés de l'emploi ou nécessitant une formation spécifique. Pour améliorer l'efficacité du dispositif, l'auteure montre que l'assortir d'une période de "protection» de l'emploi telle que tout licenciement intervenant durant cette période occasionne un remboursement de l'aide publique permet de limiter les pratiques abusives consistant à ne conserver les individus que le temps de la subvention. Les dispositifs examinés dans l'article de Moczall ont débuté en octobre 2007 dans le cadre du programme "JobPerspektive» et s'adressaient aux allocataires de l'aide sociale ayant des difficultés particulières de réinsertion dues à leur très faible qualification, leur âge élevé ou encore leur non-maîtrise de la langue allemande. L'auteur ne met pas en évidence d'effet d'aubaine ni d'effet de substitution lié à ce programme, mais il montre que les entreprises qui y recourent utilisent davantage que les autres d'autres politiques de l'emploi, ce qui renforce ainsi la compensation financière de l'État.

Enfin, les articles de Parent et Sautory et de Parent seule exposent les principaux résultats des travaux empiriques d'évaluation de l'accompagnement renforcé des demandeurs d'emploi qui constitue le cœur de la stratégie d'activation des politiques du marché du travail en Europe depuis le milieu des années 1990. Parent et Sautory s'intéressent plus particulièrement aux dispositifs d'accompagnement renforcé et au rôle des acteurs de l'accompagnement, qu'il s'agisse des conseillers du service public de l'emploi ou d'organismes privés en cas d'externalisation de l'accompagnement des demandeurs d'emploi. Si toutes les études d'évaluation répertoriées dans cet article concluent à un effet favorable du renforcement de l'accompagnement sur le retour à l'emploi des chômeurs, elles estiment souvent moins efficace le recours aux prestataires privés pour accompagner ce retour que le recours à l'opérateur public et font apparaître des résultats mitigés quant à la qualité de l'emploi retrouvé. Parent porte son attention aux dispositifs de contrôle des recherches d'emploi et de sanctions qui peuvent en découler. Les évaluations empiriques présentées convergent sur le fait que les contrôles comme les sanctions, par la menace qu'ils constituent, ont des effets positifs sur le retour à l'emploi mais négatifs sur la qualité des emplois retrouvés. Sous cette menace, les demandeurs d'emploi pourraient modifier leurs canaux de recherche, accepter des emplois moins stables et moins bien rémunérés ou même quitter le marché du travail. Afin d'éviter ces effets pervers, sans doute convient-il de doser subtilement 
l'usage et l'échelonnement dans le temps de ces mécanismes d'activation.

Ces travaux font écho à l'intervention inaugurale de David Card au colloque consacré à l'évaluation des politiques actives du marché du travail, qui ouvre ce numéro en retraçant presque quarante ans de recherche en évaluation des dispositifs de politiques actives du marché du travail en Europe et aux États-Unis. Après avoir dépeint les trois grands types d'intervention que sont l'accompagnement des demandeurs d'emploi, la formation professionnelle et les contrats aidés (ou créations directes d'emploi), Card offre un aperçu des résultats obtenus quant aux effets de ces différents dispositifs sur le retour à l'emploi des bénéficiaires selon le contexte étudié, le public ciblé et l'horizon temporel retenu. Il met de plus en parallèle à ces effets, les conséquences sur l'emploi des non bénéficiaires et les coûts supportés par l'État. Sa lecture des récentes expériences d'évaluation menées aux États-Unis nous apprend que l'orientation des chômeurs vers des dispositifs de formation repérés par les conseillers de l'agence pour l'emploi comme étant adaptés au profil du chômeur est effectivement plus efficace que le libre choix de formation donné aux demandeurs d'emploi; que le développement des aides à la création d'entreprise en réponse à des situations de licenciements économiques de grande ampleur est à la fois très coûteux pour l'État et peu rémunérateur pour les demandeurs d'emploi; enfin, qu'aucune solution durable au problème de précarité des jeunes particulièrement défavorisés ne semble clairement se dégager dans la mesure où l'amélioration observée de leur situation grâce à certains dispositifs ne persiste guère au-delà des effets de court terme.

À l'heure où les injonctions de réduction des dépenses publiques sont grandes et alors que le taux de chômage demeure élevé dans de nombreux pays européens, plusieurs questions relatives aux politiques actives du marché du travail restent encore en suspens : à quels organismes doit-on donner la responsabilité de la mise en place de ces dispositifs ? Quels sont leurs effets sur l'ensemble de la population ? Comment donner aux conseillers les moyens pour accompagner au mieux les demandeurs d'emploi ? Les travaux présentés dans ce numéro tendent d'apporter des éléments de réponse à ces questions. En ayant eu le soin de préciser le contexte dans lequel les mesures de politiques actives et leurs évaluations se sont mises en place, de présenter les effets attendus de ces mesures, les résultats antérieurement obtenus pour des dispositifs similaires et la méthode d'évaluation retenue, les auteurs de ces travaux apportent un éclairage nouveau et, nous l'espérons, utile au lecteur comme au décideur public sur le bien-fondé, ou non, des politiques actives du marché du travail.

\section{Bibliographie}

AbBring J., VAN DEN BERG G. (2003), «The non parametric identification of treatment effects in duration», Econometrica, vol. 71, n 5, pp. 1491-1517.

BAHU M. (2014), «Les contrats d'aide à l'emploi en $2012 »$, Dares analyses, $n^{\circ} 21$.

Bayardin V., Fendrich Y. (2014), «Les contrats aidés de 2005 à $2011 »$, Dares analyses, $n^{\circ} 11$.

Behaghel L., Crépon B., Gurgand M. (2009), Évaluation d'impact de l'accompagnement des demandeurs d'emploi par les opérateurs privés de placement et le programme Cap vers l'entreprise. Rapport final, septembre. Disponible en ligne à l'adresse : http://travail-emploi. gouv.fr/IMG/pdf/Rapport_Final-_CREST-ENSEE.pdf; consulté le 3 novembre 2014.

Blundell R., Costa Dias M., Meghir C., van Reenen J. (2004), «Evaluating the employment impact of a mandatory job search program », Journal of the European economic association, vol. 2, n 4, pp. 569-606.

Blundell R., Costa Dias M. (2008), «Alternative approaches to evaluation in empirical microeconomics », The journal of human resources, vol. 44, $\mathrm{n}^{\circ} 3$, pp. 565-640.
Bonnal L., Fougère D., SÉrandon A. (1997), «Evaluating the impact of French employment policies on individual labour market histories », Review of economic studies, vol. $64, \mathrm{n}^{\circ} 4$, pp. 683-713.

Boone J., Fredriksson P., Holmlund B., van Ours J. C. (2007), «Optimal unemployment insurance with monitoring and sanctions", The economic journal, vol. $117, \mathrm{n}^{\circ} 518$, pp. 399-421.

Brodaty T. (2007), «La politique active de l'emploi en faveur des jeunes : les dispositifs ont-ils touché leur cible ?», Annales d'économie et de statistique, $\mathrm{n}^{\circ} 85$, pp. 3-40.

Brodaty T., Crépon B., Fougère D. (2001), «Using matching estimators to evaluate alternative youth employment programs: evidence from France, 19861988 », in Lechner M., Pfeiffer F. (ed.), Econometric evaluations of active labor market policies in Europe, Heidelberg, Physica-Verlag, pp. 43-58.

Caliendo M. Kopeinig S. (2005), «Some practical guidance for the implementation of propensity score matching», IZA Discussion paper, $\mathrm{n}^{\circ} 1588$.

Card D., Michalopoulos C., Robins P. (2005), «When financial incentives pay for themselves: evidence from 
a randomized social experiment for welfare recipients », Journal of public economics, vol. 89, $\mathrm{n}^{\circ}$ 1, pp. 5-29.

Conseil de L'emploi, des REvenus ET DE LA COHÉSION SOCIAle (CERC) (2005), Aider au retour à l'emploi, rapport, Paris, La Documentation française.

Crépon B., Dejemeppe M., Gurgand M. (2005), «Counseling the unemployed: does it lower unemployment duration and recurrence?», IZA Discussion paper, $\mathrm{n}^{\circ} 1796$.

Direction DE L'ANIMATION DE LA RECHERChE, DES ÉTUDES ET DES STATISTIQUES (DARES) (2014), Tableau de bord des politiques d'emploi; disponible en ligne à l'adresse : http://travail-emploi.gouv.fr/ etudes-recherches-statistiques-de,76/statistiques,78/ politique-de-l-emploi-et-formation, $84 /$ tableau-de-bordmensuel-des,261/pages-de-synthese,2095.html; consulté le 3 novembre 2014 .

Dormont B., Fougère D., Prieto A. (2001), «L'effet de l'allocation unique dégressive sur la reprise d'emploi», Économie et statistique, $\mathrm{n}^{\circ} 343$, pp. 3-33.

Eissa N., Liebman J. B. (1996), «Labor supply response to the Earned Income Tax Credit», The quarterly journal of economics, vol. 111, n² 2, pp. 605-637.

Fougère D., Kamionka T., Prieto A. (2010), «L'efficacité des mesures d'accompagnement sur le retour à l'emploi », Revue économique, vol. 61, n³, pp. 599-612.

Fougère D., Kramarz F., Magnac T. (2000), «Youth employment policies in France», European economic review, vol. 44, n 4-6, pp. 928-942.

Francesconi M., Rainer H., Van Der KlaAuw W. (2009), «The effects of in-work benefit reform in Britain on couples: theory and evidence», The economic journal, vol. 119, n 535, pp. F66-F100.

Garoche B., Roguet B. (2014), «Les dépenses en faveur de l'emploi et du marché du travail en $2011 »$, Dares Analyses, $\mathrm{n}^{\circ} 18$.

Gerfin M., Lechner M. (2002), «A microeconometric evaluation of the active labour market policy in Switzerland», The economic journal, vol. 112, $\mathrm{n}^{\circ} 482$, pp. 854-893.

GIVORD P. (2010), «Méthodes économétriques pour l'évaluation de politiques publiques», Document de travail, ${ }^{\circ} \mathrm{G} 2010 / 08$, Insee.

Gomel B., Serverin É. (2013), «L'expérimentation sociale aléatoire en France en trois questions », Travail et emploi, $\mathrm{n}^{\circ} 135$, pp. 57-71.

GRITZ R. M. (1993), «The impact of training on the frequency and duration of employment», Journal of econometrics, vol. 57, $\mathrm{n}^{\circ}$ 1-3, pp. 21-51.
Heckman J. J., Ichimura H., Todd P. E. (1997), « Matching as an econometric evaluation estimator: evidence from evaluating a job training programme», The review of economic studies, vol. 64, $\mathrm{n}^{\circ}$ 4, pp. 605-654.

Heckman J. J., Ichimura H., Smith J. A., Todd P. E. (1998), «Characterizing selection bias using experimental data», Econometrica, vol. 66, n 5, pp. 1017-1098.

Heckman J. J., Lalonde R. J., Smith J. A. (1999), «The economics and econometrics of active labor market programs », in Ashenfelter O. C., Card D. (ed.), Handbook of labor economics, vol. 3, Part A., Amsterdam, Elsevier, pp. 1865-2097.

ImBens G. (2000), Efficient estimation of average treatment effects using the estimated propensity score, Econometric Society World Congress 2000, Contributed papers $n^{\circ} 1166$.

Lalive R. (2008), «How do extended benefits affect unemployment duration? A regression discontinuity approach», Journal of econometrics, vol. 142, $\mathrm{n}^{\circ} 2$, pp. 785-806.

Meyer B. D., Rosenbaum D. T. (2001), «Welfare, the Earned Income Tax Credit, and the labor supply of single mothers », The quarterly journal of economics, vol. 116, $\mathrm{n}^{\circ} 3$, pp. 1063-1114.

Organisation de COOPÉRATION ET DE DÉVElOPPEMENT ÉCONOMiQues (OCDE) (2005), Perspectives de l'emploi de l'OCDE, Paris, OCDE.

OCDE (2006), Stimuler l'emploi et les revenus, les leçons à tirer de la réévaluation de la stratégie de l'OCDE pour l'emploi, Rapport sur les orientations politiques, Paris, OCDE.

Perret B. (2008), L'évaluation des politiques publiques, Paris, La Découverte, coll. «Repères» (2e édition).

Roguet B., Schreiber A. (2009), «Les dépenses en faveur de l'emploi et du marché du travail entre 2000 et 2007 », Premières synthèses, n ${ }^{\circ}$ 52.3, Dares.

Rosenbaum P. R., Rubin D. B. (1983), «The central role of the propensity score in observational studies for causal effects », Biometrika, vol. 70, n 1, pp. 41-55.

RubiN D. B. (1974), «Estimating causal effects of treatments in randomized and nonrandomized studies », Journal of educational psychology, vol. 66, $\mathrm{n}^{\circ}$, pp. 688-701.

SiAnesi B. (2004), «An evaluation of the Swedish system of active labor market programs in the 1990s », The review of economics and statistics, vol. 86, n 1, pp. 133-155. 